\title{
A Control Strategy for Operating Unknown Constrained Mechanisms
}

\author{
Ewald Lutscher*, Martin Lawitzky**, Gordon Cheng* and Sandra Hirche**
}

\begin{abstract}
This work aims at the development of a versatile control strategy for operating unknown mechanically constrained devices such as drawers or doors. Few assumptions on the device's shape as well as the utilized hardware are required. Our approach is based on an on-line estimation of the constraint manifold which serves as a reference input for an admittance-type controller providing the compliance required. The direction estimation is obtained from the velocity signal in task space. An on-line adaptation of the admittance controller according to the estimated moving direction reduces contact forces. The functionality of the control strategy is demonstrated on a mobile manipulator in a kitchen environment.
\end{abstract}

\section{INTRODUCTION}

In recent years, robotics research spread out from the specialized area of industrial plants into everyday human environments, which are designed for human abilities. The integration of robots into human environments is desirably arranged seamlessly without major modifications to the environment itself. Many tasks in human environments require the manipulation of simple mechanisms such as doors, drawers or lockers. Robots must be able to operate them in a general manner like any human can do. Actuating such mechanisms is not always affordable, and in fact, human-like abilities is one of the key aspects of humanoid robotics.

A major problem arises from the wide range of the devices described above, which make model-based approaches difficult to apply. There exists a large variety of opening arcs, handles, and general techniques to operate such mechanisms. Besides, the device to operate is not always unresisting. Magnets, springs or tight gaskets keep drawers and respectively doors closed and need a certain amount of force to apply. The only property all those devices have in common is their restriction of movement to one-DoF manifolds. A drawer can only be pulled or pushed along one direction just as a door swings along a certain arc. A human does not need to know the position of the hinge or the pushing/pulling direction. Instead he just tries to push or pull and lets the structure guide the rest of the motion. Unlike human, very few robotic systems have taken advantage of this fact.

The philosophy behind our approach is to provide an algorithm which is very general in its applicability, hence makes as few assumptions as possible. A robotic arm which has contact with the operated object is presumed to avoid

\footnotetext{
* Ewald Lutscher and Gordon Cheng are now with the Institute for Cognitive Systems (ICS), Faculty of Electrical Engineering, Technische Universität München, D-80290 München, Germany ewald.lutscher@tum.de, gordon.cheng@ieee.org ** Martin Lawitzky and Sandra Hirche are with the Institute of Automatic Control Engineering (LSR), Faculty of Electrical Engineering, Technische Universität München, D-80290 München, Germany \{ml, hirche\}etum.de
}

contact loss as well as being capable of generating the required forces to move the device. The focus here is on the device manipulation and we neglect the reaching/grasping task. The only sensory feedback is provided by a force-torque sensor at the wrist and joint angle measurements for position control what is most common in robotics. The manipulator is mounted on an omnidirectional mobile base which supports the execution of the manipulation task. Based on this we are going to show a universal way of operating unknown constrained mechanisms.

\section{A. Related Work}

Most of the existing approaches focus only on a specific task like opening a door and rely on several planning algorithms, which involves at least a minimal model of the device being operated. Nagatani and Yuta introduce a strategy for opening doors that makes use of an analytical description of the door handle trajectory [8], [9]. Peterson et al. use in [12] a minimal analytical model of a door for which the parameters are estimated in combination with a predictive controller. However, they only achieve a $90 \%$ success rate even with known relative positions. Pujas et al. [15] used a hybrid position-force control to open a door. This kind of strategies suffer in general from modelling and localization errors, which are likely to occur. A behavior based approach is pursued in [19] and [5] with the focus on the entire task of opening and traversing a door. They either assume a known door-frame [19] or do not make fixated contact with the door [5] to simplify the subtask of opening. Probabilistic methods, known from autonomous mobile robotics, are introduced in [13] to solve manipulation tasks. Again, a model of the door based on laser data is presumed. Other works make use of special hardware components to fulfill the manipulation task. Schmid et al. [17] introduced a spatial tactile sensor matrix obtained from a modified gripper in combination with a force-torque-sensor at the wrist. In [16], only tactile feedback from a multi-fingered hand is used and in [6] new methods for obtaining the contact force dispensing completely with the classical wrist sensor are proposed. The DLR lightweight robot possesses a sophisticated position, torque and impedance control system that can be used to open doors [1]. A force follow behavior is implemented for their lightweight manipulator combined with a mobile base, which drives through a door. Such passive types of controllers are inappropriate for tasks where a force and hence active action of the manipulator is required. An approach which aims at a general method for operating constrained mechanisms can be found in [14]. In this work, the task frame formalism is used to represent the relative pose of the 
end-effector and task frame. However, detailed information how this approach can applied to specific problems is not given. The approach presented here is in parts inspired by the idea of choosing the path of least resistance [10]. For that neither a an exact mechanism model nor absolute position information is required. In [10] direct force input is used implying knowledge about the forces necessary to operate the mechanism. Furthermore, neither end-effector orientation nor mobile manipulation are addressed, which is included in our approach.

\section{B. General Idea}

Our strategy for operating constrained mechanisms is adopted from human behavior, for example when opening a door in a dark room. Despite the hinge location is unknown it is usually no problem for a human to find the direction of movement by kinesthetic exploration of the mechanism. One starts pulling or pushing in one direction and lets the structure guide any further motions. This strategy permits the operation of various devices with the only restriction that it has only one degree of freedom.

The significant uncertainty which arises with such a general approach brings along an urgent need for compliant control of the manipulator to imitate the passive compliance of a human. Compliant motion control algorithms were addressed in several works, see e.g. [2], [3]. It has been shown that the impedance control framework introduced by Hogan [4] allows good performance, while preserving stability and robustness. It is also a convenient tool for handling impact situations and to cope with unknown environments. A derivation from the classical impedance control is presented by the admittance control [20] where the impedance loop is closed around a motion controller.

In the present work we will show how an admittance control framework can be exploited to find the path of least resistance and reduce contact forces when operating unknown constrained mechanisms. The outcome is a very general and unified approach, which is applicable to any robot which meets a minimum of hardware requirements.

The remainder of the paper is structured as follows: Section II will give an overview of the underlying control algorithm and explain in detail the proposed approach. In Section III the practical implementation and experimental evaluation will be presented.

\section{MAnipulation CONTROL}

\section{A. Admittance Control Scheme}

In the following the applied admittance control scheme is presented, first for the translational DoFs and later for the orientation. A translational mechanical impedance states a mapping from the position deviation $\Delta \boldsymbol{p}=\left(\Delta p_{x}, \Delta p_{y}, \Delta p_{z}\right)^{T}$ to the force $f$ according to the second-order dynamics

$$
\boldsymbol{f}=\boldsymbol{M}_{v} \Delta \ddot{p}+\boldsymbol{D}_{v} \Delta \dot{p}+\boldsymbol{K}_{v} \Delta p
$$

where the $3 \times 3$ matrices $\boldsymbol{M}_{v}, \boldsymbol{D}_{v}$ and $\boldsymbol{K}_{v}$ denote the virtual inertia, damping and stiffness matrix describing the desired target impedance. Note that by convention bold characters are used for vectors and matrices. Likewise an admittance maps an applied force to a position deviation by solving the differential equation (1). The classical admittance control scheme for a robotic manipulator takes a desired trajectory $\Phi_{d}(t)$ given as position $\boldsymbol{p}_{d}(t)$, velocity $\dot{\boldsymbol{p}}_{d}(t)$ or acceleration $\ddot{\boldsymbol{p}}_{d}(t)$ commands and maps it according to (1) to a compliant trajectory $\Phi_{c}(t)$ with the compliant commands $\boldsymbol{p}_{c}(t), \dot{\boldsymbol{p}}_{c}(t)$, $\ddot{p}_{c}(t)$ where

$$
\Delta \boldsymbol{p}(t)=\boldsymbol{p}_{c}(t)-\boldsymbol{p}_{d}(t)
$$

We propose a velocity-based admittance controller as there exists no a priori knowledge about the forthcoming trajectory. The desired velocity is determined by a desired movement direction $\boldsymbol{d}_{d}$ and a specified constant velocity magnitude $\nu_{d}$ by $\dot{\boldsymbol{p}}_{d}=\nu_{d} \boldsymbol{d}_{d}$ where $\left|\boldsymbol{d}_{d}\right|=1$. The constrained mechanisms considered in this paper can be represented by a 1-DoF manifold. Ideally, the desired direction of motion $\boldsymbol{d}_{d}$ is in the tangent space of this manifold, i.e. is equal to the actual movement direction of the manipulator

$$
d=\frac{\dot{p}}{|\dot{p}|} \text {. }
$$

In application noisy measurements of $\dot{p}$, however, prevent the direct use of $d$. Instead a filtered version will be used as explained in Section II-C. The compliant velocity command $\dot{\boldsymbol{p}}_{c}(t)$ is computed from (2) where $\Delta \boldsymbol{p}(t)$ is computed by solving (1).

During free space motion no external forces are present $\boldsymbol{f}=\mathbf{0}$, hence the admittance relation does not modify the desired trajectory $\Phi_{d}=\Phi_{c}$. When a contact occurs $\boldsymbol{p}_{d}$ can be interpreted as an attractor point which penetrates the constraint-surface causing contact forces $f$ according to (1). The end-effector behaves like a mass-spring-damper system with the parameters $\boldsymbol{M}_{v}, \boldsymbol{D}_{v}$ and $\boldsymbol{K}_{v}$.

When $\boldsymbol{p}_{d}$ traverses the constraint-surface the difference between desired and actual position $\boldsymbol{\Delta}=\boldsymbol{p}_{d}-\boldsymbol{p}$ can be decomposed into a component parallel $\boldsymbol{\Delta}_{\|}$to the actual constraint-surface and a component $\boldsymbol{\Delta}_{\perp}$ perpendicular to it causing the contact forces $\boldsymbol{f}_{\|}$and $\boldsymbol{f}_{\perp}$. Each component together with its derivative and second derivative is used in an admittance relation like (1). If no friction is present then $\boldsymbol{f}_{\|}=\mathbf{0} \Rightarrow \Delta \boldsymbol{p}_{\|}=\mathbf{0} \Rightarrow \boldsymbol{p}_{d \|}=\boldsymbol{p}_{c\|\|}$.

The contact force $\boldsymbol{f}_{\perp}$ results in a translational deviation which is $\Delta \boldsymbol{p}_{\perp}=-\boldsymbol{\Delta}_{\perp}$ and can be computed by solving (1). Thus the perpendicular component is completely suppressed so that the effective translation is executed solely along $\boldsymbol{\Delta}_{\|}$. For small $\boldsymbol{\Delta}$ the end-effector follows the path of least resistance for any trajectory without explicitly computing or knowing it. This effect of the admittance control can be exploited to operate constrained mechanisms in a very natural way.

\section{B. Control of the Attractor Point}

When choosing a constant $\boldsymbol{p}_{d}$, the operated mechanism will surely move, assuming the correct hemisphere for the initial movement has been set. However, the contact forces will potentially grow as the attractor point will penetrate further and further away from the constraint-surface. Hence, 
a continuous adaptation of the motion direction via the commanded velocity should be made.

The basic idea of the proposed algorithm is the following, see also Fig. 1 for a visualization:

1) Induce an initial translational motion of the endeffector $\nu_{d} \boldsymbol{d}_{0}$

2) Determine continuously the resulting movement direction $d$

3) Set $\dot{\boldsymbol{p}_{\boldsymbol{d}}}=\nu_{d} \boldsymbol{d}$

The normalized vector $\boldsymbol{d}_{0}=\boldsymbol{d}_{d}(t=0)$ denotes the initial movement direction set by the user or some higher-level algorithm. For determining the initial desired movement direction only knowledge of the approximate direction (hemisphere) of opening or closing the mechanism is required. The admittance controller will immediately force the endeffector on the path of least resistance. The occurring small deviations of $\Phi_{d}$ from $\Phi_{c}$ together with low stiffness $\boldsymbol{K}_{\boldsymbol{v}}$ result in only small contact forces.

Many devices in human environments need a certain amount of force to move them or initialize the movement. This will cause the end-effector to remain in its initial position before the force needed to operate the device is applied along the trajectory space of $\Phi_{c}$. As a result the attractor point may depart from the constraint-surface before the motion direction can be reliably estimated. In this case, even when the true direction of motion is obtained, an offset between the velocity-controlled attractor point $p_{d}$ and the constraint trajectory $\Phi_{c}$ will remain resulting in persistent contact forces $f_{\perp}$. In order to reduce the persistent contact forces we propose to modify the admittance control law using a projection approach. Specifically, the deviation $\Delta p$ is projected onto the actual direction of motion $\boldsymbol{d}$ according to

$$
\Delta \hat{\boldsymbol{p}}=\frac{1}{|\boldsymbol{d}|^{2}} \boldsymbol{d}^{T} \Delta \boldsymbol{p d}
$$

where $\Delta \hat{\boldsymbol{p}}$ replaces $\Delta \boldsymbol{p}$ in (1), completing the proposed approach for the translational DoF.

Note that the coupling between the translational and rotational DoF prohibits to use the same approach for translation and orientation. Here we propose to adopt a force-follow scheme for the orientation with a zero-stiffness admittance control

$$
\boldsymbol{m}=\boldsymbol{M}_{v \mathrm{rot}} \dot{\boldsymbol{\omega}}+\boldsymbol{D}_{v \mathrm{rot}} \boldsymbol{\omega}
$$

where $\boldsymbol{\omega}$ the angular velocity, $\boldsymbol{M}_{\text {vrot}}$, and $\boldsymbol{D}_{v \text { rot }}$ the $3 \times 3$ virtual inertia and damping matrices, and $\boldsymbol{m}$ the measured

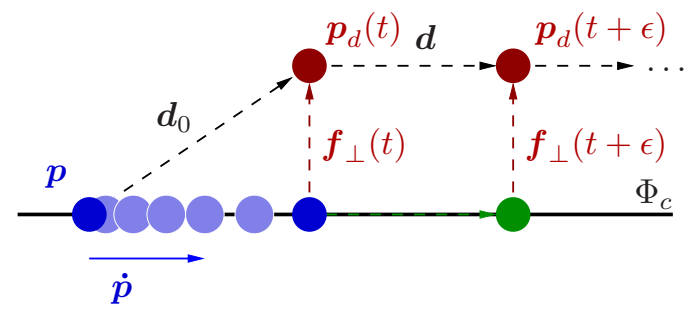

Fig. 1. Illustration of the proposed scheme without projection: Although the direction of motion is correctly estimated, an offset remains between $\boldsymbol{p}_{d}$ and $\Phi_{c}$ generating $\boldsymbol{f}_{\perp}$.

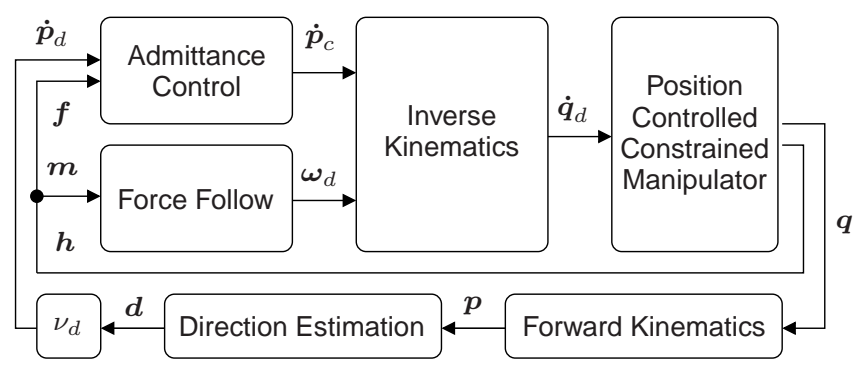

Fig. 2. Admittance control scheme. The six dimensional wrench $\boldsymbol{h}=\left(\boldsymbol{f}^{T}, \boldsymbol{m}^{T}\right)^{T}$ denotes the forces and moments measured at the endeffector.

end-effector torque.

As a result, the translational branch is controlled with a standard admittance relation (1) while a force-follow behaviour is implemented for the rotational part with (4). The resulting twist

$$
\dot{\boldsymbol{x}}_{d}=\left[\begin{array}{c}
\dot{\boldsymbol{p}}_{c} \\
\boldsymbol{\omega}_{d}
\end{array}\right]=\left[\dot{p}_{x}, \dot{p}_{y}, \dot{p}_{z}, \omega_{x}, \omega_{y}, \omega_{z}\right]^{T}
$$

is transformed to the corresponding desired joint velocity vector $\dot{\boldsymbol{q}}_{d}$ using instantaneous inverse kinematics. The desired joint angle $\boldsymbol{q}_{\boldsymbol{d}}$ is obtained through integration of $\dot{\boldsymbol{q}}_{d}$ and serves as the input for the innerloop joint angle controller.

A PD joint-controller for each joint assures fast and stable tracking of the desired joint angles

$$
\boldsymbol{\tau}=\boldsymbol{D}_{j}\left(\dot{\boldsymbol{q}}_{d}-\dot{\boldsymbol{q}}\right)+\boldsymbol{P}_{j}\left(\boldsymbol{q}_{d}-\boldsymbol{q}\right)
$$

where the diagonal matrices $\boldsymbol{D}_{j}$ and $\boldsymbol{P}_{j}$ contain the controller gains for each individual joint, and $\tau$ denotes the commanded motor torques. The proposed overall control scheme is visualized in the block diagram in Fig. 2.

\section{Estimating the Path of Least Resistance}

Typically, the velocity measurement takes place in joint space via differentiation of the position signal from the encoders, i.e. it is prone to measurement and quantization noise. The forward kinematics and the normalization step further increases the noise level. In order to reduce the noise level it is proposed to estimate the moving direction using a simple moving average (SMA) filter in the velocity domain. Here the SMA is applied to every component of the velocity measurement $\dot{\boldsymbol{p}}_{m}$ of $\dot{\boldsymbol{p}}$ separately resulting in the filtered velocity signal

$$
\dot{\boldsymbol{p}}_{\text {filt }}(t)=\frac{1}{T_{M A}} \int_{t-T_{M A}}^{t} \dot{\boldsymbol{p}}_{m}(\tau) d \tau
$$

where $T_{M A}$ characterizes the time window over which the SMA is taken. The filtered signal $\dot{p}_{\text {filt }}$ is normalized to acquire the estimation of the movement direction

$$
\boldsymbol{d}_{\mathrm{filt}}=\frac{\dot{\boldsymbol{p}}_{\mathrm{filt}}}{\left|\dot{\boldsymbol{p}}_{\mathrm{filt}}\right|},
$$

which effectively represents the estimate of the tangent vector of the constraint manifold and replaces $\boldsymbol{d}$ in (3). 


\section{Control of Base Movement}

A stationary manipulator has a highly limited workspace. Even a common task, like opening a cupboard door can not be accomplished for such a constricted robot. An extension of the workspace can be achieved by mounting the manipulator on a mobile platform. The manipulators workspace is basically limited by external obstacles, its maximum reach, joint limits and collision with itself or the mobile base.

Important for the success of the proposed manipulation controller is that all platform movements do not affect the end-effector pose in the world frame, i.e. any platform movement must be cancelled out by an appropriate endeffector movement. Due to space limitations the coupling control algorithm cannot be presented here. The platform control itself is based on existing schemes for self collision and joint limit avoidance and are not discussed in detail, see [7] for details.

\section{EXPERIMENTAL EVALUATION}

\section{A. Hardware Setup}

The proposed method is implemented on an omnidirectional mobile platform equipped with two identical humanscaled 7-DoF arms, see [18] for details which are frontmounted in a mirrored configuration to provide a human-like work space. Forces and torques are measured using a six-axis $J R 3$ force-torque sensor. The joint angles are measured by digital MR-encoders with a resolution of 4096 counts per revolution. A two-finger gripper is mounted at each wrist with rubber glued to the inside of the fingers preventing slippage of the grip and adding at least a minimum of mechanical compliance. The platform, see [11] for details, is equipped with four conventional wheels. An omnidirectional design provides human-like maneuverability.

\section{B. Control Implementation}

The control modules of the arms are implemented with Simulink in combination with the Real-Time Workshop using the Real-Time Application Interface for Linux (RTAI). A static kinematic model is computed at runtime using the software tool Autolev and the velocity-based admittance controller proposed in Section II is implemented using forcetorque measurements of the JR3 sensor. The admittance

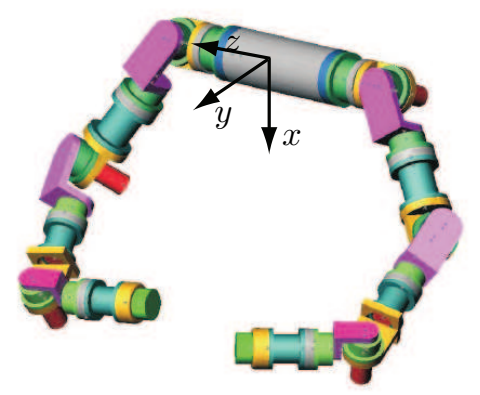

Fig. 3. Robotic manipulator with reference frame [18]. controller runs with $1 \mathrm{kHz}$. Furthermore, the estimation algorithm and the indicated platform control are implemented. As an additional measure for noise reduction, a deadzone is implemented for the measurements $\dot{\boldsymbol{p}}_{m}$ resulting in welldefined values of $\boldsymbol{d}_{f i l t}$ also for very small velocities.

\section{Experiments and Results}

In the following the general performance and effects of some of the parameters of our approach is examined. Exemplarily operated mechanisms are a cupboard door, a drawer and a microwave door providing linear (drawer) as well as arced constraint manifolds with different hinge locations. The cupboard door and the microwave door have in addition the property that they need a certain amount of force $(20 \mathrm{~N}$ and $15 \mathrm{~N}$, respectively) before they start moving without any noteworthy resistance. The robot is always placed roughly in front of the device. The initial movement direction is set to $\boldsymbol{d}_{0}=\left(0, \sin \left(-\frac{\pi}{4}\right), \sin \left(-\frac{\pi}{4}\right)\right)^{T}$ resulting in a large initial error angle of approximately $45^{\circ}$. The desired opening speed $\nu_{d}$ is randomly varied between $0.01 \frac{\mathrm{m}}{\mathrm{s}}$ and $0.05 \frac{\mathrm{m}}{\mathrm{s}}$, the moving average window for the SMA is chosen out of the interval $T_{M A} \in[0.3,1.0] \mathrm{s}$. The admittance parameters have been chosen heuristically so that no contact instability occured; a systematic design procedure is subject to future work. Under these conditions all of the performed tests (20 for each device) have been successful, see Fig. 4 for snapshots, i.e. we observe a superior success rate of $100 \%$.
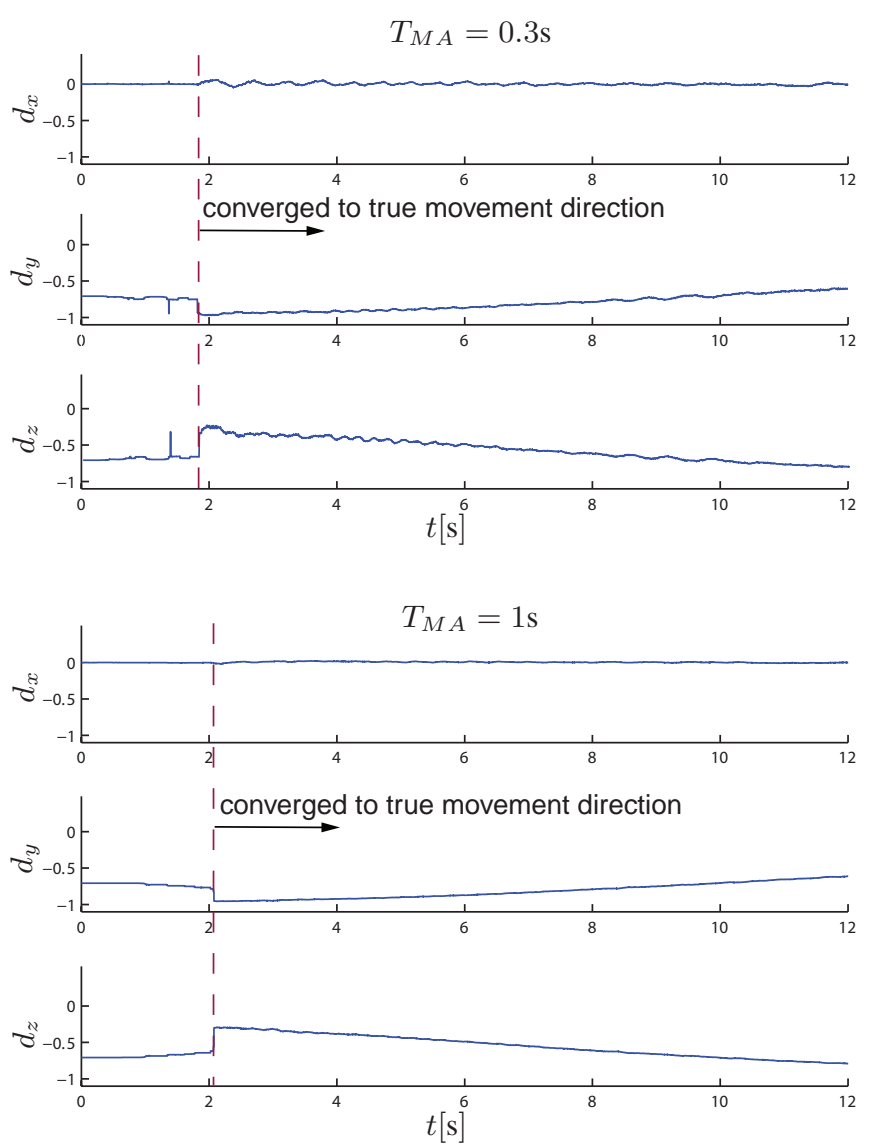

Fig. 5. Effect of moving average window $T_{M A}$ during opening of cupboard door: Improved noise reduction, but higher phase lag for higher $T_{M A}$. 

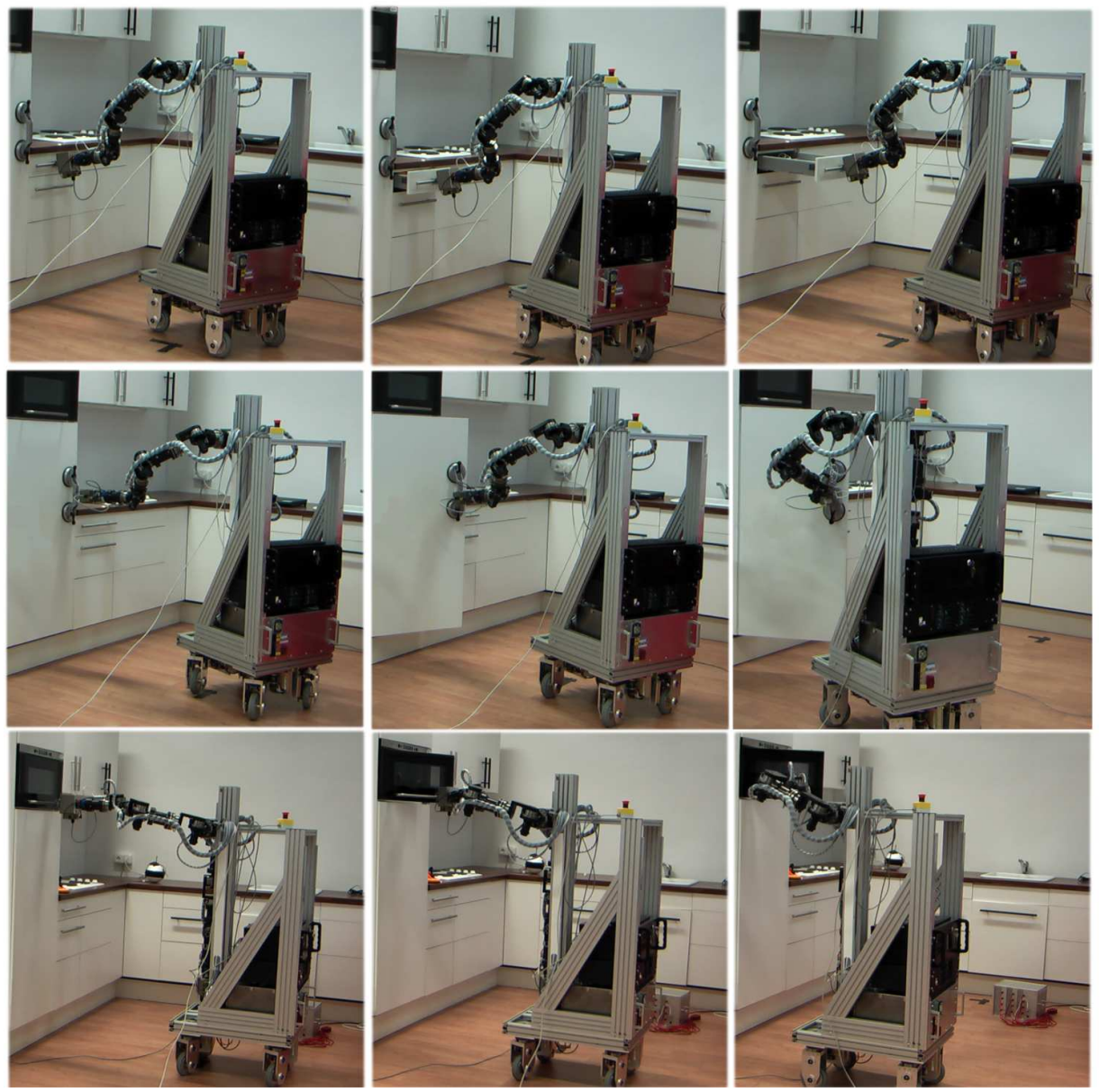

Fig. 4. Mobile manipulator operating drawer, cupboard and microwave door using the propsed control strategy without change of parameters.

The influence of the SMA filtering can be observed in Fig. 5 where estimation process over time for two different time windows is shown for the opening of the cupboard door. For the sake of clarity, the here presented measurements are collected without a moving base. Note that the estimation converges quickly to the true direction motion. As expected, a larger window leads to a smoother direction estimation. However, we also observe a larger phase lag which potentially may result in increased contact forces especially at high velocities and/or mechanisms with high curvature.

Finally, in order to validate the proposed projection approach (3) the opening of the drawer with the initial resisting force is performed. The initial movement direction $\boldsymbol{d}_{0}$ is again set to $\left(0, \sin \left(-\frac{\pi}{4}\right), \sin \left(-\frac{\pi}{4}\right)\right)^{T}$. This results in an increase of $\boldsymbol{f}_{\|}=\left(0, f_{y}, 0\right)$ and $\boldsymbol{f}_{\perp}=\left(0,0, f_{z}\right)$ with approximately the same slope as can be observed from Fig. 6 . When the break-away force is reached $\boldsymbol{f}_{\|}$vanishes quickly while $f_{\perp}$ still remains even though the direction estimation has converged to the true movement direction $\boldsymbol{d}$. As a result, the contact force applied in $y$-direction remains nearly $20 \mathrm{~N}$. Applying the modification of the admittance relation (1) according to (3), leads to disappearance of the persistent contact force, as shown in Fig. 7 validating the proposed approach.

\section{CONCLUSION}

In this paper, a novel manipulator control strategy for operating unknown constrained one-DoF mechanisms such as doors or drawers is proposed. The approach is based on a modified admittance control scheme. The enabling innovation here is the estimation of the tangent along the constraint manifold and the according modification of the desired path for the admittance control scheme. As a result arbitrary one-DoF mechanisms can be operated without requiring a model and without changing the control parameters. In addition to the high versatility of the proposed approach it poses also only moderate hardware requirements: any admittance controlled manipulator mounted on an omnidirectional mobile platform is suitable. Outstandingly, the control strategy is also suitable to operate mechanisms with a holding spring, there is no need to modify the operated mechanisms in any way (removing springs or other resisting devices). The performed experiments demonstrate the superior robustness of the approach with respect to different constrained mechanisms and initial conditions. Future work includes the improved estimation of the movement direction and the extension to chained mechanisms. 

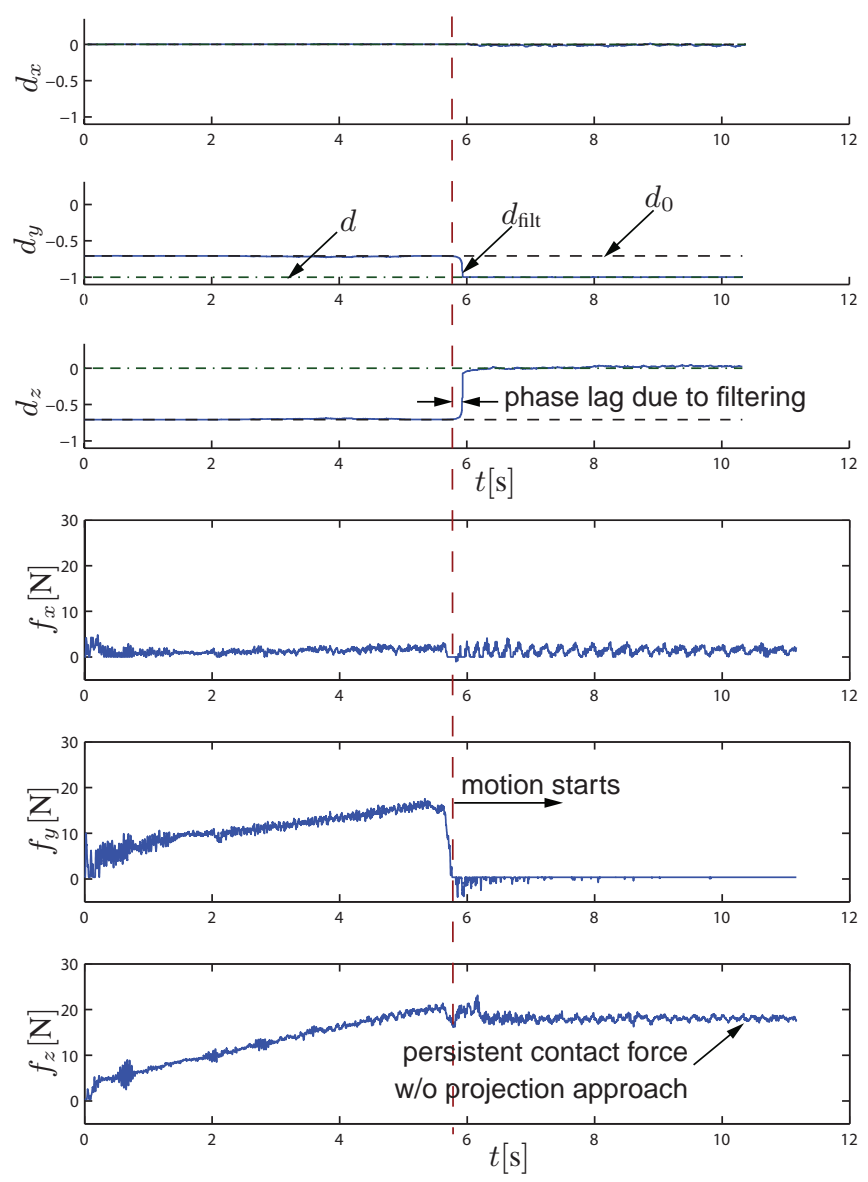

Fig. 6. Direction estimation and contact force for opening drawer with breakaway force and initialization error of approx. $45^{\circ}$ without projection approach.
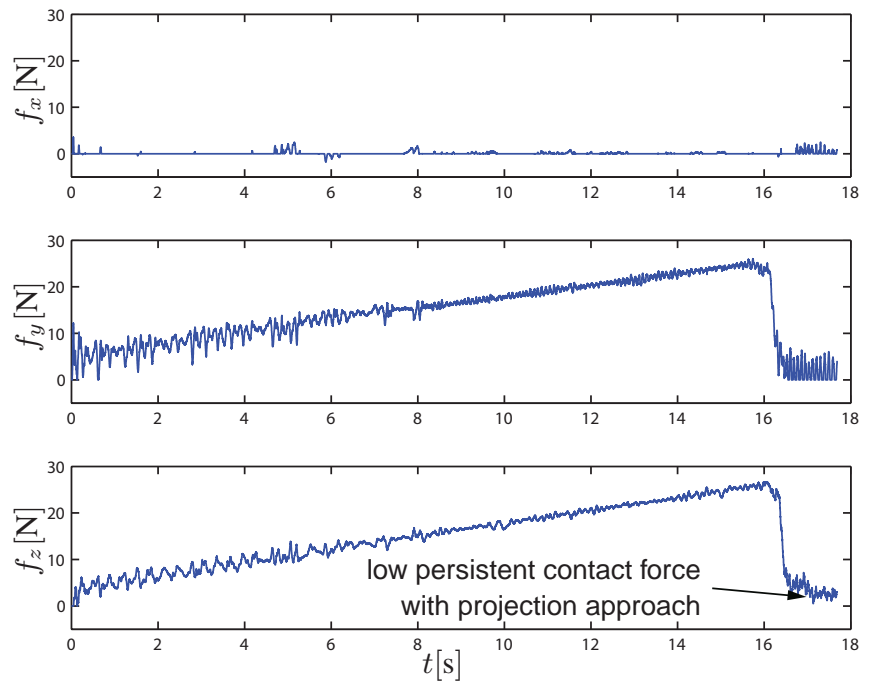

Fig. 7. Contact force for opening drawer with breakaway force and initialization error of approx. $45^{\circ}$ with projection approach.

\section{ACKNOWLEDGEMENTS}

This work is supported in part within the DFG excellence initiative research cluster Cognition for Technical Systems CoTeSys (www. cotesys.org).

\section{REFERENCES}

[1] A. Albu-Schaffer, C. Ott, and G. Hirzinger. A Unified Passivity-based Control Framework for Position, Torque and Impedance Control of Flexible Joint Robots. The International Journal of Robotics Research, 26(1), 2007.

[2] J. De Schutter and H. Van Brussel. Compliant Robot Motion I. A Formalism for Specifying Compliant Motion Tasks. The International Journal of Robotics Research, 7(4), 1988.

[3] J. De Schutter and H. Van Brussel. Compliant Robot Motion II. A Control Approach Based on External Control Loops. The International Journal of Robotics Research, 7(4), 1988.

[4] N. Hogan et al. Impedance Control: An Approach to Manipulation, Part I-III. ASME Journal of Dynamic Systems, Measurement and Control, 107, 1985

[5] A. Jain and C.C. Kemp. Behaviors for Robust Door Opening and Doorway Traversal with a Force-Sensing Mobile Manipulator.

[6] J. Kang, C. Hwang, and G. Park. A Simple Control Method for Opening a Door with Mobile Manipulator. In ICCAS, 2003.

[7] O. Khatib. Real-time obstacle avoidance for manipulators and mobile robots. The International Journal of Robotics Research, 5(1), 1986.

[8] K. Nagatani and S. Yuta. An Experiment on Opening-Door-Behavior by an Autonomous Mobile Robot with a Manipulator. In 'Human Robot Interaction and Cooperative Robots', Proc. on 1995 IEEE/RSJ International Conference on Intelligent Robots and Systems, volume 2, 1995.

[9] K. Nagatani and S. Yuta. Designing strategy and implementation of mobile manipulator controlsystem for opening door. In Proc. of IEEE International Conference on Robotics and Automation, volume 3, 1996.

[10] G. Niemeyer and J.J.E. Slotine. A simple strategy for opening an unknown door. In Proceedings on the IEEE International Conference on Robotics and Automation, 1997., volume 2, 1997.

[11] N. Nitzsche and G. Schmidt. A mobile haptic interface mastering a mobile teleoperator. In Proceedings on the IEEE/RSJ International Conference on Intelligent Robots and Systems, 2004. (IROS 2004), volume 4.

[12] L. Peterson, D. Austin, and D. Kragic. High-level control of a mobile manipulator for door opening. In Proceedings on the IEEE/RSJ International Conference on Intelligent Robots and Systems, 2000. (IROS 2000), volume 3, 2000.

[13] A. Petrovskaya and A.Y. Ng. Probabilistic Mobile Manipulation in Dynamic Environments, with Application to Opening Doors. In International joint conference on artificial intelligence, (IJCAI'07), Hyderabad, 2007

[14] M. Prats, S. Wieland, T. Asfour, A.P. del Pobil, and R. Dillmann. Compliant interaction in household environments by the Armar-III humanoid robot. In 8th IEEE-RAS International Conference on Humanoid Robots, 2008., 2008.

[15] A. Pujas, P. Dauchez, and F. Pierrot. Hybrid position/force control: task description and control schemedetermination for a real implementation. In Proceedings on the IEEE/RSJ International Conference on Intelligent Robots and Systems, 1993. (IROS 93), volume 2, 1993.

[16] C. Rhee, W. Chung, M. Kim, Y. Shim, and H. Lee. Door opening control using the multi-fingered robotic hand for the indoor service robot. In Proceedings on the IEEE International Conference on Robotics and Automation, 2004. (ICRA'04), volume 4, 1926.

[17] A.J. Schmid, N. Gorges, D. Goger, and H. Worn. Opening a door with a humanoid robot using multi-sensory tactile feedback. In ICRA 2008. IEEE International Conference on Robotics and Automation, 2008.

[18] B. Stanczyk. Development and Control of an Anthropomorphic Telerobotic System. $\mathrm{PhD}$ thesis, Technische Universität München, 2006.

[19] BJW Waarsing, M. Nuttin, and H. Van Brussel. Behaviour-based mobile manipulation: the opening of a door. Proc. of ASER'03, Bardolino, Italy, 2003.

[20] D.E. Whitney. Force Feedback Control of Manipulator Fine Motions. In Trans. ASME, J. DSMC, vol.99, no.2, 1977. 